\title{
Cesf-740104--4
}

Note: This is a draft of a paper being submitted for Contents of thts baper stoud not be quoted nor referred to wi thout permisston of the althor $(s)$.

\section{REMARKS ON THE THEORY OF FAST NEUTRON SPUTTERING}

\author{
Mark. T. Robinson
}

SOLID STATE DIVISION

OAK RIDGE NATIONAL LABORATORY

Operated by

UNION CARBIDE CORPORATION

for the

U. S. ATOMIC ENERGY COMMISSION

Oak Ridge, Tennessee, 37830

\section{January 1974}


REMARKS ON THE THEORY OF FAST NEUTRON SPUTTERING*

Mark T. Robinson

Solid State Division, Dak Ridge National Laboratory

$P$. O. Box $X$, Oak Ridge, Tennessee 37830

\section{Abstract}

Theoretical estimates of neutron sputtering yields are in serious disagreement with experiment, unlike the situation with ion sputtering. Possible reasons for the discrepancy are sought without success. It is shown that chunk ejection by neutrons is not due to single neutron events nor to the dynamic interference of cascades. The need for more complete experimental data to guide development of the theory is emphasized.

\section{Introduction}

Predictions of the rates of erosion of solids by fast neutron irradiation $(1,2)$ have been based on the sputtering theory of sigmund (2). These estimates are not in agreement with experimental observations. It is the purpose of this paper to explore a few of the possible reasons for these rather puzzling discrepancies. Theoretical estimates of the neutron sputtering yields of four metals are listed in Table 1. These

TABLE 1

Neutron Sputtering Yields Calculated from the Sigmund Theory

\begin{tabular}{|c|c|c|c|}
\hline \multirow{2}{*}{ Target } & \multirow{2}{*}{$U_{0}(e V)$} & \multicolumn{2}{|c|}{ Yield (atoms $/ 10^{5}$ neutrons) } \\
\hline & & 235 U Fission & D-T Fusion \\
\hline Al & 3.33 & 2.4 & 4.2 \\
\hline $\mathrm{Cu}$ & 3.50 & 1.9 & 6.0 \\
\hline Nb & 7.56 & 0.89 & 2.7 \\
\hline $\mathrm{Au}$ & 3.80 & 1.1 & 4.0 \\
\hline
\end{tabular}

are based on the sigmund theory and apply to spherical targets immersed in an isotropic flux of neutrons. Published calculations (3) of the energy deposited by fast neutrons were used. The primary recoil energies were corrected for inelastic atomic scattering by the method of Lindhard (4). Leachman's (5) form of the ${ }^{235} \mathrm{U}$ fission spectrum was

"Research sponsored by the U. S. Atomic Energy Commission under contract with Union Carbide Corporation. 
used. The heats of sublimation were taken from Gschneider (6). As far as they exist, the experimental neutron sputtering data are assembled in Table 2 . The observations are

TABLE 2

Experimental Neutron Sputtering Yields

\begin{tabular}{|c|c|c|c|}
\hline Target & Neutron Source & Yield (atoms $/ 10^{5}$ neutrons) & Reference \\
\hline \multirow[t]{6}{*}{$\mathrm{Au}$} & $D-T$ & 300 & Garber (9) \\
\hline & & $<60$ & Keller (11) \\
\hline & ${ }^{235} U$ fission & 10 & Anno (12) \\
\hline & & 0.2 & Verghese (14) \\
\hline & & 500 & Blewitt (8) \\
\hline & $\mathrm{Pu}-\mathrm{Be}$ & $5 \times 10^{4}$ & Keller (10) \\
\hline $\mathrm{Fe}$ & ${ }^{235} \mathrm{U}$ fission & 600 & Anno (13) \\
\hline Nb & D-T & $(2.5 \pm 1) \times 10^{4}$ & Kaminsky (7) \\
\hline
\end{tabular}

very scattered, so much so that some doubt must be expressed that even the magnitude of the yield is known. Nevertheless, it is likely that the predicted yields are much too low. While some of the discrepancy can be assigned to geometrical effects, since the experimental targets were not spherical and were not usually irradiated isotropically, most of it must be due to inadequacies in the theory.

Sigmund's theory (2) is based on the concept of a displacement cascade which underlies all radiation damage theory. He calculates the energy deposited in a thin region near the target surface as the displacement cascade initiated by the primary recoil spreads out, excluding any energy lost by electron excitation. Aside from geometrical factors, the sputtering yield is just the ratio of the deposited energy to the binding energy of surface atoms, this being taken as the heat of sublimation of the target. Sigmund compared his theory with the existing data on reflection sputtering (the ordinary kind) of polycrystalline metals by ions with energies from $10^{2}$ to $10^{6} \mathrm{eV}$. Further tests of the theory have been made by Andersen and Bay $(15,16)$. The agreement between theory and experiment is generally excellent. It is observed, however, that the yields measured for heavy ions, in the energy region where these are maximal, are often considerably greater than predicted. This is exemplified by the case of $10^{5} \mathrm{eV} \mathrm{Xe}^{+}$ions on $\mathrm{Cu}$, where the discrepancy reaches about $30 \%$. To test the possibility that these discrepancies are due to nonlinear effects occurring in high density cascades, Andersen and Bay (16) compared the yields observed with monatomic and diatomic ions of the same velocity. The yields per incident atom were found to be significantly larger in the latter

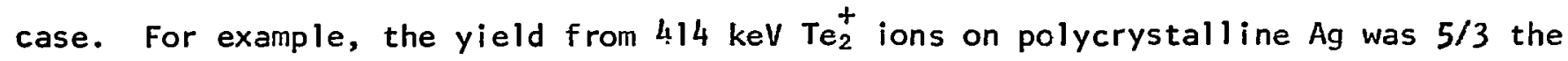


yield from $207 \mathrm{keV} \mathrm{Te}^{+}$. The experiment with the molecular ions represents an extreme example of overlapping cascades and shows the importance of cascade nonlinearities. The nature of the nonlinearity is not known. It could involve, for instance, a cooperative overcoming of the surface binding forces whan many adjacent atoms are set into motion at about the same time. A possibly related topic is the observation that sputtered ions often appear in rather large clusters (17), containing as many as ter or twelve atoms. Attempts to produce sputtered molecular species in computer simulations have so far proved unsuccessful (18).

Another cilass of experiment which seems not to be comprehended by the Sigmund theory is concerned with the temperature dependence of sputtering yields. Although the yields from polycrystalline metals are usually considered temperature indeperdent, it has been observed $(19,20)$ that as the target temperature approaches the melting point, the yield may rise as much as a factor 10. This occurs over a range of $100 \mathrm{~K}$ or more. Thompson and Nelson (19) ascribe the additional sputtering to the evaporation of atoms by thermal spikes. Experiments with 25 to $45 \mathrm{keV} \mathrm{Ne}^{+}, \mathrm{Ar}^{+}$, and $\mathrm{Xe}^{+}$ions on several metals (20) lead to spike radi $i$ near $100 \AA$, lifetimes of a few picoseconds, and spike temperature rises of a few hundred degrees. Such a thermal spike is also a cascade nonlinearity and may well be very similar to that mentioned above. The description in thermal terms is not unique and should not be taken too literally, especially since the spike lifetimes are so short that the concept of temperature is not really applicable. Nonetheless, the thermal spike approach provides a method for dealing, however clumsily, with cooperative ejection of particles from a solid surface.

\section{Dynamic Interference Between Cascades}

Kaminsky's original experiment (7) on the $14 \mathrm{MeV}$ neutron sputtering of $\mathrm{Nb}$ was characterized by the observation that the ejected material appeared in large chunks. Simple arguments on energetics make it plain that single neutron hits cannot be responsible for the ejection of such chunks. A typical chunk may be regarded as a sphere of radius 1 um. Such a sphere contains about $2.3 \times 10^{11}$ atoms altogether, of which about $1.8 \times 10^{8}$ are in the surface. The minimum energy for producing such an object is probably its surface energy. That is, we imagine the chunk to be quarried out of the irradiated foil, the only cost being creation of the associated new surface. The free surface energy of $\mathrm{Nb}$ is about $0.18 \mathrm{eV}$ per atom (21). Thus, at least $32 \mathrm{MeV}$ would be required to eject such a chunk, an amount far in excess of that available from $14 \mathrm{MeV}$ neutron hits on $\mathrm{Nb}$, where the maximum recoil energy is about $600 \mathrm{keV}$. Thus, single neutron effects cannot be responsible for the ejection of such chunks. 
The possibility that two or more cascades may interfere and thus produce nonlinear effects is easily calculated: Assign a characteristic dimension L to each displacement cascade, such that sputtering occurs only if the cascade is within $L$ of a surface. The rate at which such cascades occur, per unit area of the target surface, is no $\phi L$, where $n$ is the atomic density of the target, $\sigma$ is its neutron cross-sectioin, and $\phi$ is the flux density of the incident neutrons. The neutron hits are distributed in space and time according to Poisson statistics. Thus, the probability that one or more neutrons will make collisions in a volume $V$ during a time $t$ is

$$
1-\exp (-n \sigma \phi V t) \approx n \sigma \phi V t
$$

as long as the collision probability is small. For two cascades to interfere, $V$ must be approximately $L^{3}$ and $t$ must be the lifetime of a cascade. Thus, the rate at which interferences occur is

$$
B=(n \sigma \phi)^{2} L^{4} \tau
$$

where $\tau$ is the cascade lifetime.

The quantities $L$ and $\tau$ can be estimated from simple considerations. The former may be regarded as the total pathlength traversed by the primary recoil. Fig. I shows estimates of $L$ for $N$ recoils in Nb. These are taken from the work of Schiatt (22) who

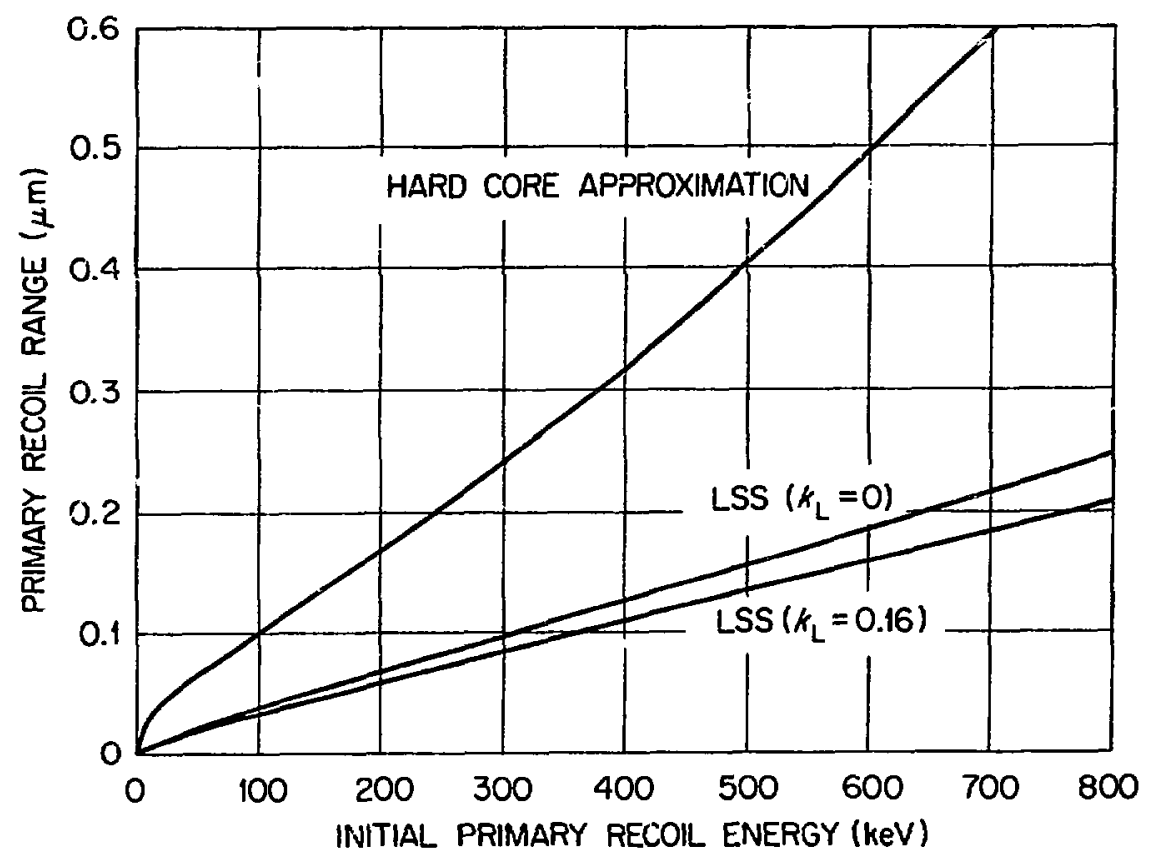

FIG. 1

Total Path Lengtn Calculated for Nb Recoils in Nb. 
used the well-known iSS theory (23) and from the hard-core approximation calculation of Holmes and Leibfried (24), using an exponentially screened Coulomb potential to describe the atomic scattering. The two LSS curves are for no inelastic energy loss $\left(k_{L}=0\right)$ and for an amount of inelastic loss based on Lindhard's theory of electronic stopping $(4,23)$. The LSS estimates are the more reliable, but the hard core results set a strong upper limit. Fig. 2 shows similar estimates of the time necessary for the primary to slow down from its initial energy to various final energies, $E_{f}$. This time is approximately the time necessary to produce the entire cascade. The Holmes-Leibfried treatment of $L$ is easily modified to estimate the slowing down tire (25). Another estimate can be obtained

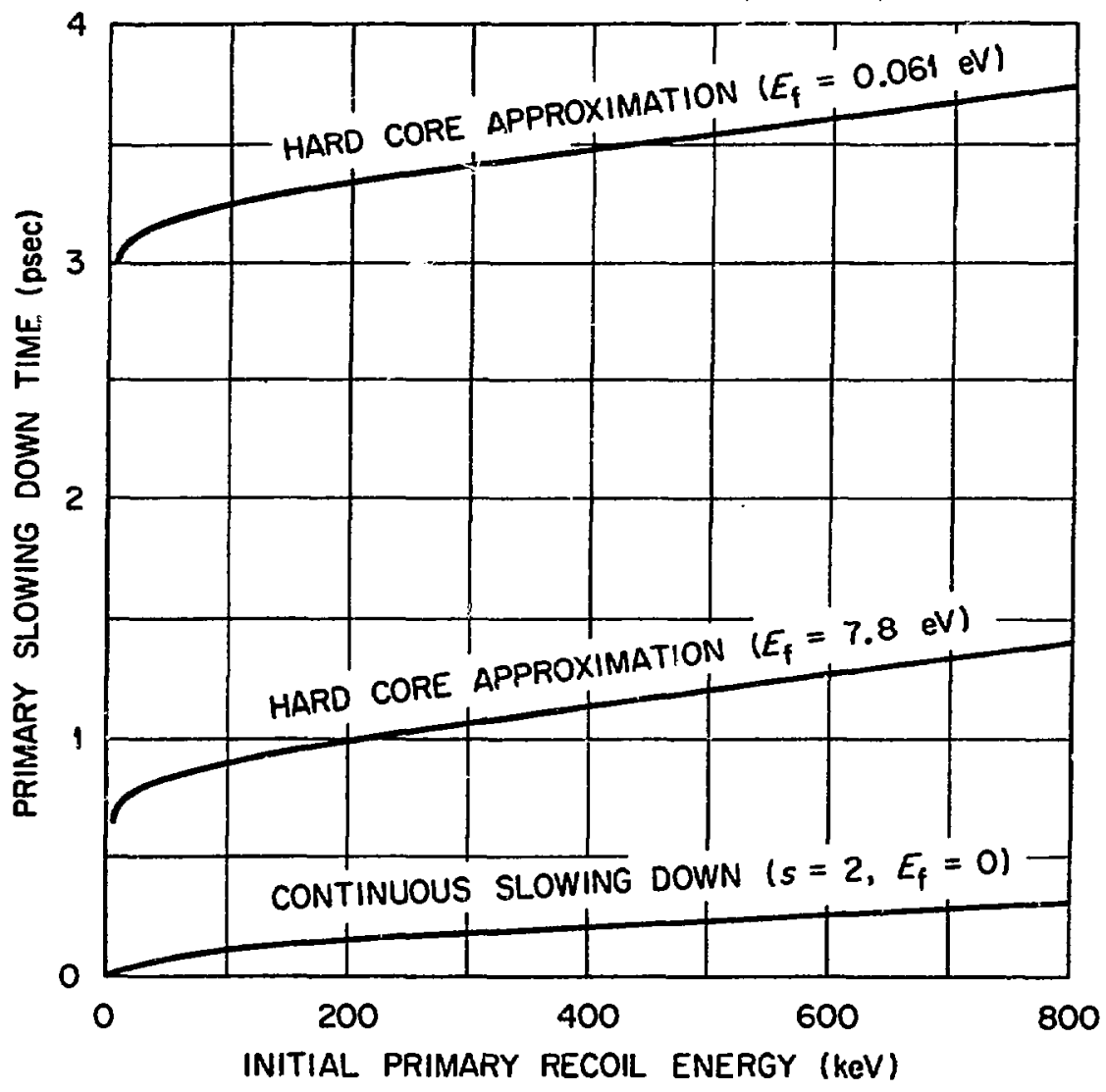

FIG. 2

Slowing Down Times Calculated for Nb Recoils in Nb.

by integrating the nuclear stopping power using Lindhard's treatment (26) of an $r^{-2}$ potential. Again the hard core results set a strong upper limit.

From Figs. I and 2, we take for $600 \mathrm{keV} \mathrm{Nb}$ recoils the values $L=0.45 \mu \mathrm{m}$ and $\tau=$ 3.6 psec. With $\sigma=3.91$ barns and $\phi=2.4 \times 10^{10} \mathrm{n} / \mathrm{cm}^{2} \mathrm{sec}$, the interference rate is $B=3.8 \times 10^{-10} \mathrm{~cm}^{-2} \mathrm{sec}^{-1}$. In 54 hours, the number of interferences is only $7 \times 10^{-5}$ $\mathrm{cm}^{-2}$, to be compared with the observed (7) density of chunks, around $10^{3} \mathrm{~cm}^{-4}$. Thus, 
dynamic interferences between cascades cannot accounc for the ejection of chunks. It must be concluded that the ejection of chunks in neutron sputtering is primarily associated with aspects of the target which are not considered in displacement cascade production theories, such as details of microstructure.

\section{Thermal Spikes and Neutron Sputtering}

Although the thermal spike concept has not been a very fruitful one in radiation damage, the success of Thompson and Nelson $(19,20)$ in applying it to the temperature dependence of ion sputtering makes it worth examining in the context of neutron sputtering. We imagine a thermal spike to be a sphere of radius $L$ which persists for a time $\tau$. Within the sphere and during its 1 ifa, the atoms are heated to a uniform temperature $T_{s}$. If one of these spheres should intercept the target surface, atoms would evaporate from the surface. The flux dersity of atoms evaporating from a plane surface at a temperature $T$ is

$$
f(T)=4 n(k T / 2 \pi m)^{1 / 2} \exp \left(-U_{0} / k T\right)
$$

where $k$ is Boltzmann's constant, $m$ is the mass of the evaporating atoms, and $U_{0}$ is the binding energy of atoms in the target surface. This result is obtained by integration of the evaporation energy spectrum given by Thompson and Nelson (19). The spike temperature can be associated with the energy of an atom recoiling from a neutron interaction by

$$
E=2 \pi L^{3} n k\left(T_{s}-T_{0}\right)
$$

where $T_{0}$ is the temperature of the target. Assuming for simplicity that the neutrons are monoenergetic and that the recoiling atoms are uniformly distributed in energy, the neutron sputtering yield can be written as

$$
s=n \sigma \int_{0}^{E_{m}} f\left(T_{s}\right)\left(d E / E_{m}\right) \tau \int_{0}^{L} \pi\left(L^{2}-x^{2}\right) d x
$$

where $T_{s}$ is defined by Eq. (2), $E_{m}$ is the maximum possible recoil energy and $x$ is the distance of a thermal spike below the target surface. If the target temperature is neglected and if the spike parameters $L$ and $\tau$ are taken as constants, Eq. (3) can be written as

where

$$
\begin{gathered}
S=\frac{8 n \sigma E_{m} \tau}{3\left(2 \pi m U_{0}\right)^{1 / 2}}\left[y^{4} \int_{y}^{\infty} e^{-u^{2}} \frac{d u}{u^{4}}\right], \\
y=\left[2 \pi L^{3} n U_{0} / E_{m}\right]^{1 / 2} .
\end{gathered}
$$


The function of $y$ in Eq. (4) can be evaluated in terms of tabulated functions. We note here only that it has a maximum value of about 0.1 when $y$ is about 0.5 .

To compare Eq. (4) with the measurements of Blewitt, et al. (8) on Au, assume that $2 \mathrm{MeV}$ neutrons typify the ${ }^{235} \mathrm{U}$ fission spectrum. Then, with $\mathrm{E}_{\mathrm{m}}=40 \mathrm{keV}$ and $\sigma=6.4$ barns, we must have $s<6 \times 10^{-5} \tau$, where $\tau$ is measured in psec. Since the lifetime of a cascade will probably not exceed about $5 \mathrm{psec}$, yields in excess of about $3 \times 10^{-4}$ would not be expected. At the same time, to obtain a value of $y$ near 0.5 requires $L$ to be about $20 \AA$. From Schiatt's tables (22), the range of Au recoils in Au is about $90 \AA$ at $40 \mathrm{keV}$ and about $60 \AA$ at $20 \mathrm{keV}$. Thus, the thermal spike model used here requires spikes that are smaller (and therefore hotter) than expected. This could merely reflect the assumption that the spikes are spherical. The model could be modified to consider spikes which were cylinders or prolate ellipsoids with major dimension corresponding to the primary recoil range. While this calculation has not been carried through, it could give reasonable cascade dimensions, although it would probably require lifetimes greater than is plausible. Finally, application of Eq. (4) to $14 \mathrm{MeV}$ neutron sputtering of $\mathrm{Nb}$ gives $\mathrm{S}<$ $5 \times 10^{-4} \tau$ (psec) and $L$ about $40 \AA$, the latter to be compared with the much larger figures in Fig. 1. Here the neglect of inelastic energy losses in cascade development must be significant. It also seems possible that subcascade formation at the high energies of these $\mathrm{Nb}$ recoils might need to be considered.

\section{Discussion}

While the thermal spike treatment has some possibility of accounting for neutron sputtering yields greater than those given by the Sigmund theory, it should be clear that no real theoretical understanding of neutron sputtering yet exists. This depressing state of affairs cannot easily be rectified by tinkering with the essentials of cascade production theory, of defect migration theory, or the like, since changes in these areas would have important implications in the theory of ion sputtering, where agreement between theory and experiment is satisfactory. However, it must be said that the experimental ion sputtering data are generally in accord among different workers, something which Table 2 shows not to be the case in neutron sputtering. Before much useful theory can be done in the latter field, it will be necessary to improve greatly the experimental situation, to the extent that one can say with reasnnable certainty what the yields are in particular cases.

The remarks made here have certain implications for research on fusion devices. The gerieral lack of theoretical understanding of neutron sputtering is so complete that one must consider that it may be strongly influenced by almost any other accompanying influence on the CTR first wall. Thus, alteration of the microstructure of the wall by 
the formation of blisters, voids, or gas bubbles, roughening of the surface by ion sputtering, and thermal effects must all be considered of potential significance in neutron erosion pheromena. Thus, many complicated experiments involving these variables must be contemplated, at least until some reasonable understanding has been achieved.

I wish to express my appreciation to R. Behrisch, D. P. Jackson, L. H. Jenkins, K. Meríle, 0. S. Oen, J. E. Robinson, and F. W. Young, Jr., for many stimulating discussions of neutron sputtering. I am grateful to T. H. Blewitt and to M. Kaminsky for communicating their results in advance of this conference, in addition to several discussions.

\section{References}

1. R. Behrisch, Nuclear Fusion 12, 695 (1972).

2. P. Sigmund, Phys. Rev. 184, 383 (1969).

3. M. T. Robinson in Nuclear Fusion Reactors (British Nuclear Energy Society, London, 1970), P. 364 .

4. J. Linchard, V. Nielsen, M. Scharff, and P. V. Thomsen, Kgl. Danske Videnskab. Selskab, Mat.-Fys. Medd. 33, No. 10 (1963).

5. R. B. Leachman, Proc. First Intern. Conf. Peaceful Uses Atomic Erergy, Geneva 2, 193 (1955).

6. K. Gschneider, Solid State Physics 16, 344 (1964).

7. M. Kaminsky and S. K. Das, Proceedings of this Conference.

8. T. H. Blewitt, M. Kirk, A. C. Klank, R. Malewicki, and T. Scott, ibid.

9. R. 1. Garber, G. P. Dolya, V. M. Kolyada, A. A. Modlin, and A. 1. Fedorenko, Zhur. Eksp. Teor. Fiz. Pis'ma Z, 375 (1968) [English translation: JETP Letters Z, 296 (1968)].

10. K. Keller and R. V. Lee, Jr., J. Appl. Phys. 37, 1890 (1966).

11. K. Keller, Plasma Physics 10, 195 (1968).

12. D. W. Norcross, B. P. Fairand, and J. N. Anno, J. Appl. Phys. 37, 621 (1966).

13. T. S. Bair and J. N. Anno, J. App 1. Phys. 43, 2453 (1972).

14. K. Verghese, Trans. Am. Nuciear Soc, 12, 544 (1969).

15. H. H. Anderseri and H. Bay, Rad. Effects 13, 67 (1972).

16. H. H. Andersen and H. L. Bay, Rad. Effects 19, 139 (1973).

17. G. Staudenmaier, Rad. Effects 13, 87 (1972).

18. D. P. Jackson, Atomic Energy of Canada Limited, private communication. 


\section{References (Continued)}

19. M. W. Thompson and R. S. Nelson, Phil. Mag. $\underline{7}, 2015$ (1962).

20. R. S. Neison, Phil. Mag. $I_{2} 291$ (1965).

21. B. C. Allen, Trans. Metal. Soc. AIME 227, 1175 (1963).

22. H. E. Schiatt, Kgl. Danske Videnskab. Selskab, Mat.-Fys. Medd. 35, No. 9 (1966).

23. J. Lindhard, M. Scharff, and H. E. Schiatt, ibid. 33, No. 14 (1963).

24. D. K. Holmes and G. Leibfried, J. Appl. Phys. 31, 1046 (1960).

25. M. T. Robinson, unpublished calculation.

26. J. Lindhard, V. Nielsen, and M. Scharff, Kgl. Danske Videnskab. Selskab, Mat.-Fys. Medd. 36, No. 10 (1968). 